\title{
On the Longwave Climate Feedbacks
}

\author{
YI HUANG \\ Department of Atmospheric and Oceanic Sciences, McGill University, Montreal, Quebec, Canada
}

(Manuscript received 10 January 2013, in final form 12 March 2013)

\begin{abstract}
This paper mainly addresses two issues that concern the longwave climate feedbacks. First, it is recognized that the radiative forcing of greenhouse gases, as measured by their impact on the outgoing longwave radiation (OLR), may vary across different climate models even when the concentrations of these gases are identically prescribed. This forcing variation contributes to the discrepancy in these models' projections of surface warming. A method is proposed to account for this effect in diagnosing the sensitivity and feedbacks in the models. Second, it is shown that the stratosphere is an important factor that affects the OLR in transient climate change. Stratospheric water vapor and temperature changes may both act as a positive feedback mechanism during global warming and cannot be fully accounted as a "stratospheric adjustment" of radiative forcing. Neglecting these two issues may cause a bias in the longwave cloud feedback diagnosed as a residual term in the decomposition of OLR variations. There is no consensus among the climate models on the sign of the longwave cloud feedback after accounting for both issues.
\end{abstract}

\section{Introduction}

The climate sensitivity parameter $S$ predicts the firstorder change in global mean surface temperature $T_{s}$ in response to a radiative forcing $F$ [see the review by Ramaswamy et al. (2001)]. In general, this relationship can be expressed as

$$
\Delta R=F+S^{-1} \Delta T_{s}
$$

Here $R$ denotes the radiation flux at the top of the atmosphere (TOA). From a diagnostic point of view, the value of $S$ can be thought to be determined by the overall effect of a variety of "feedbacks" (see appendix A). Here, a feedback refers to a physical quantity $X$ other than forcing that influences $R$, and the strength of the feedback $\Delta R_{X}$ can be assessed by the partial contribution of this factor to the change in $R$. The kernel method (Soden et al. 2008; Shell et al. 2008) is a widely used method for analyzing feedback strength. In this method, a noncloud feedback is approximated by multiplying a precalculated first-order sensitivity kernel $\partial R / \partial X$ and a climate response $\Delta X$ :

Corresponding author address: Yi Huang, 805 Sherbrooke Street West, Montreal, QC H3A 0B9, Canada.

E-mail: yi.huang@mcgill.ca

$$
\Delta R_{X}=\frac{\partial R}{\partial X} \Delta X
$$

The cloud feedback can then be assessed as the residual change in $R$. Alternatively, Zelinka et al. (2012) provide a method that uses cloud property histograms to independently assess cloud feedback, although this method requires additional computation of cloud optical properties.

This paper mainly addresses two issues concerning the longwave feedbacks in transient climate change, particularly those analyzed with the kernel method. The first concerns the forcing $F$. In the "scenario experiments" [e.g., A1B, A2, etc. in the fourth Intergovernmental Panel on Climate Change (IPCC) report; Solomon et al. 2007] in which well-mixed greenhouse gas concentrations are specified, $F$ is considered to be equally set across different models, so that model discrepancies in $T_{s}$ projections are primarily attributed to their differences in climate sensitivity $S$. Reflecting this view, in kernel method-based feedback analyses, $F$ is usually assumed to be a constant value [e.g., Soden et al. (2008) use an estimate of $4.3 \mathrm{~W} \mathrm{~m}^{-2}$ over the twenty-first century in the A1B experiment, adopting the value from the third IPCC report]. Ignored in such a view, however, is the fact that the absorptions of greenhouse gases overlap with each other and with clouds and thus $F$, as measured by TOA radiation fluxes (in $\mathrm{W} \mathrm{m}^{-2}$ ), is also 
dependent on nonforcing species (e.g., atmospheric water vapor and cloud). As water vapor and cloud climatology and projection differ noticeably in different models, $F$ may differ as well. There is evidence that greenhouse gas forcing may substantially differ in different models (Soden et al. 2008). Ignoring this effect may exaggerate the intermodel differences in climate sensitivity $S$ when attributing $T_{s}$ projection differences. This error may also propagate to cloud feedback if it is not independently assessed. To recognize and account for this effect, a modification to the kernel method is proposed in this paper.

The second issue concerns the stratospheric effect on the outgoing longwave radiation (OLR). It has been recognized for a long time that the stratosphere cools when anthropogenic $\mathrm{CO}_{2}$ warms the surface-troposphere climate. In numerical experiments, when $\mathrm{CO}_{2}$ concentration is instantly and drastically increased it is observed that the stratosphere cools and steadies much faster than the surface-troposphere system equilibrates and the stratospheric changes induce a small (relative to the direct radiative effect of doubling $\mathrm{CO}_{2}$ ) change in the TOA radiation energy budget. This justifies treating stratospheric effect on the radiation energy budget as an adjustment in radiative forcing (Hansen et al. 1997). However, when transient (nonequilibrium) climate change (such as in reality and in many scenario experiments) is of interest, multiple issues may invalidate this treatment. First, in a scenario in which greenhouse gases change gradually but continuously, the system does not equilibrate and both the direct (instantaneous reduction of OLR) and indirect (via cooling the stratosphere) radiative effects of the gases may vary with time. In fact, the diagnosis of Huang (2013) shows that stratosphere has a strong time-variant effect on the OLR. Second, certain stratospheric radiative species, such as water vapor, may change in response to the change in tropospherestratosphere transport that can be linked to surface temperature and thus act as a feedback mechanism. To examine the stratosphere's role in transient climate change, the radiative effects of stratospheric temperature and water vapor variations on OLR are explicitly evaluated using radiative kernels in this paper.

In the following sections, a global warming experiment that was used in previous feedback analyses is revisited, in order to examine the two issues raised above; and then the paper is concluded with a discussion section.

\section{Feedbacks}

For the interest in this paper, the change in OLR is partitioned into the following terms:

$$
\begin{aligned}
\Delta R= & F+\Delta R_{P}+\Delta R_{L}+\Delta R_{W_{t r}}+\Delta R_{T_{s t}}+\Delta R_{W_{s t}} \\
& +\Delta R_{C}+Z
\end{aligned}
$$

The terms on the RHS are the OLR changes caused by greenhouse gas forcing $F$, by vertically uniform temperature change at the surface and throughout the troposphere (the Planck effect) $\Delta R_{p}$, by vertically nonuniform tropospheric temperature change (the lapse rate feedback) $\Delta R_{L}$, by tropospheric water vapor change $\Delta R_{W_{\mathrm{tr}}}$, by stratospheric temperature change $\Delta R_{T_{\mathrm{st}}}$, by stratospheric water vapor change $\Delta R_{W_{\mathrm{st}}}$, and by cloud change $\Delta R_{C}$. The residual term $Z$ accounts for the uncertainty in the forcing, as well as the nonlinear terms that are left out when the feedbacks are estimated using linear kernels according to Eq. (2).

Here, the linear trend in the global mean OLR in the first 50 years of the Special Report on Emissions Scenarios (SRES) A1B experiment from the Coupled Model Intercomparison Project, phase 3 (CMIP3) archive is analyzed to understand how the OLR trend results from the forcing and longwave feedbacks as decomposed in Eq. (3). There are 18 models that submitted necessary data for analyzing the longwave feedbacks. The noncloud feedbacks are computed by multiplying the kernels of Shell et al. (2008) with the linear trends in monthly mean temperature and water vapor as simulated by each GCM. The tropopause is set to linearly increase from $100 \mathrm{hPa}$ at the equator to $300 \mathrm{hPa}$ at the poles following the previous analyses (e.g., Soden and Held 2006; Soden et al. 2008; Shell et al. 2008). The cloud feedback is then obtained by using the cloud forcing adjustment (CFA) method (Soden et al. 2008; Shell et al. 2008), which combines with Eq. (3) the clear-sky OLR decomposition:

$$
\begin{aligned}
\Delta R^{\mathrm{clr}}= & F^{\mathrm{clr}}+\Delta R_{P}^{\mathrm{clr}}+\Delta R_{L}^{\mathrm{clr}}+\Delta R_{W_{\mathrm{tr}}}^{\mathrm{clr}}+\Delta R_{T_{\mathrm{st}}}^{\mathrm{clr}} \\
& +\Delta R_{W_{\mathrm{st}}}^{\mathrm{clr}}+Z^{\mathrm{clr}} .
\end{aligned}
$$

If neglecting the residual terms $-\left(Z-Z^{\text {clr }}\right)$, one obtains

$$
\Delta R_{C}=\left(\Delta R-\Delta R^{\mathrm{clr}}\right)-\left(F-F^{\mathrm{clr}}\right)-\sum\left(\Delta R_{X}-\Delta R_{X}^{\mathrm{clr}}\right) .
$$

Table 1 shows the ensemble mean and standard deviation of the noncloud feedback components analyzed with the kernel method [Eq. (2)]. The values of the tropospheric feedbacks (Planck, lapse-rate, and tropospheric water vapor) closely reproduce those reported by Held and Shell [2012, their Eq. (16)] based on the same experiment despite the fact that different periods 
TABLE 1. Breakdown of the noncloud feedbacks. Unit: $\mathrm{W} \mathrm{m}^{-2} \mathrm{~K}^{-1}$. The WV stands for water vapor.

\begin{tabular}{lrcccc}
\hline & Planck & Lapse rate & WV-tropo & T-strat & WV-strat \\
\hline Mean & -3.07 & -0.72 & 1.95 & 0.22 & 0.07 \\
Std & 0.05 & 0.24 & 0.21 & 0.11 & 0.02 \\
\hline
\end{tabular}

are analyzed (the whole twenty-first century in Held and Shell; the first 50 years of the century here).

\section{Forcing variation}

If one assumes that the forcing $F$ in all the models in the A1B experiment is a constant of $4.3 \mathrm{~W} \mathrm{~m}^{-2}$ by the end of the twenty-first century as Soden et al. (2008) do, and that half of it is realized in the first 50 years, and if one further uses an empirical relationship between the all-sky and clear-sky forcing strengths (Soden et al. 2008),

$$
\frac{F^{\mathrm{clr}}}{F}=1.16,
$$

then the cloud feedback can be obtained using Eq. (5).

However, when the noncloud and cloud feedback values are substituted back to Eq. (3), considerable residuals are discovered (see Fig. 1). The magnitudes of these residuals (an ensemble mean of $1.23 \mathrm{~W} \mathrm{~m}^{-2}$ ) are apparently nonnegligible, compared to the overall OLR changes $\left(0.70 \mathrm{~W} \mathrm{~m}^{-2}\right)$. The magnitude of the residuals is so big that they cannot be simply attributed to the nonlinear effect, which should be very small as shown by many previous studies (e.g., Soden and Held 2006; Huang et al. 2007; Shell et al. 2008; Soden et al. 2008; Huang et al. 2010). Moreover, there is a substantial spread (a standard deviation of $0.47 \mathrm{~W} \mathrm{~m}^{-2}$ ) of the residuals across these models; also, a significant correlation (a correlation coefficient of 0.55 ) exists between these residuals and global mean surface temperature changes. The evidence here indicates that a substantial portion of forcing that varies from model to model is unaccounted for and left as residual in the above feedback analysis.

An alternative way to supplement forcing information in feedback analysis is to estimate the value of $F$ in each model. A fact that one can take advantage of is that the residual term in the decomposition of the clear-sky OLR change is rather small (no more than a few percent even when highly spectrally resolved OLR is considered) (Huang et al. 2010; see appendix B for an explanation) when forcing is accurately known. Using Eq. (4), one can first estimate the clear-sky forcing $F^{\mathrm{clr}}$ as the overall change in the clear-sky OLR minus the sum of

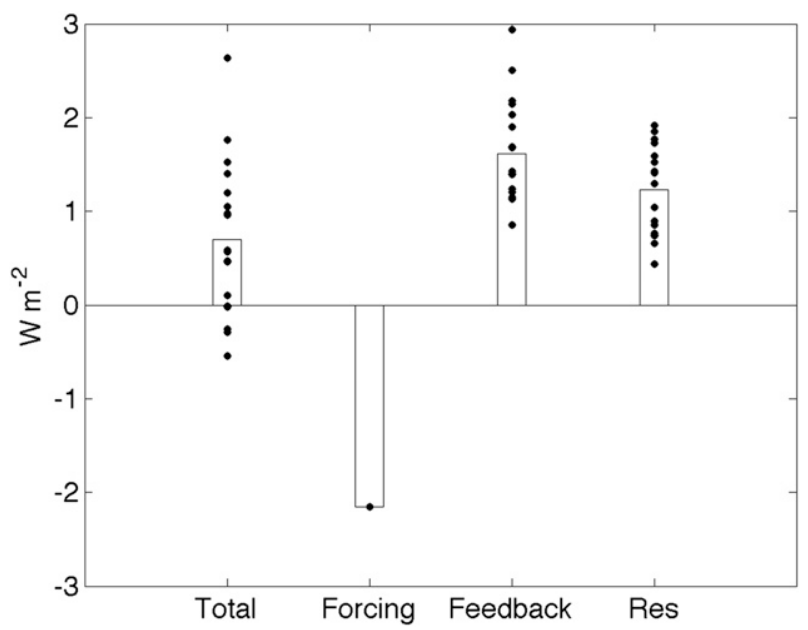

FIG. 1. Decomposition of $\triangle$ OLR. The overall change in OLR (Total) is simulated by the GCMs; the forcing is assumed to be a constant value; the feedback is the sum of noncloud and cloud feedbacks calculated using Eqs. (2) and (5), respectively; the residual term (Res) is then obtained using Eq. (3). Each dot represents a model and the bar denotes the multimodel mean.

noncloud feedbacks obtained using kernels, and then the all-sky forcing $F$ using Eq. (6).

Using the alternative way introduced above does not change the noncloud feedbacks that are assessed by the kernels but changes the overall longwave sensitivity parameter $S^{-1}$ evaluated as $\left[\Delta T_{S} /(\Delta R-F)\right]^{-1}$ based on Eq. (1) and the cloud feedback evaluated using Eq. (5). As Fig. 2 shows, ignoring the $F$ difference in different models may cause 1) an overestimate (an increase of about $25 \%$ ) of intermodel spread of the sensitivity, which results from the considerable residuals that are not explained in the previous analysis; and 2) a bias in the cloud feedback, which is seen from Table 2 (case $\mathrm{C} 1$ ) and demonstrated by Fig. 3. It is interesting to note that a similar bias (overestimate in the positiveness of longwave cloud feedback) resulted from using the CFA

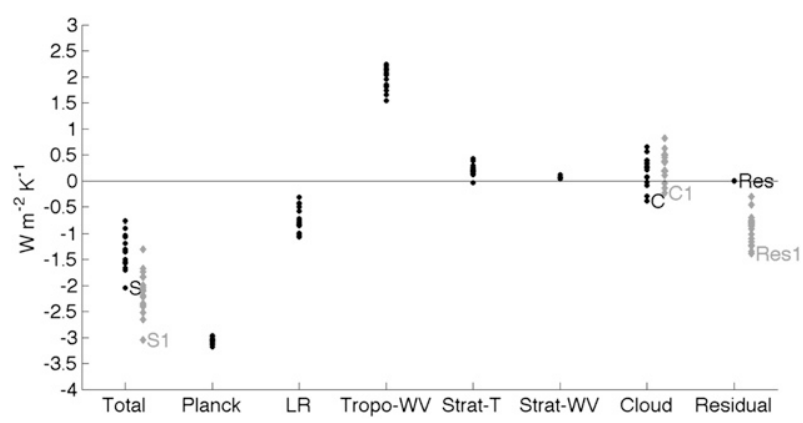

FIG. 2. Decomposed longwave climate sensitivity. Black dots denote the climate sensitivity parameter (Total) and feedbacks analyzed when forcing $F$ is estimated for each model. Gray diamonds denote the results obtained when $F$ is assumed constant. 
TABLE 2. Total, tropospheric, stratospheric, and cloud feedbacks. For the four cloud feedback estimates, C0 is assessed using the CFA method while considering the forcing variation across the models (using the method proposed in this paper) and the stratospheric effects; $\mathrm{C} 1$ is assessed using the CFA method while considering the stratospheric effects but assuming a constant forcing across the models; $\mathrm{C} 2$ is assessed using the CFA method while considering the forcing variation but ignoring the stratospheric effects; and C3 is calculated as a residual using Eq. (3) while considering the forcing variation but ignoring the stratospheric effect. Unit: $\mathrm{W} \mathrm{m}^{-2} \mathrm{~K}^{-1}$.

\begin{tabular}{lrrccccc}
\hline \hline & Total & Tropo & Strat & Cloud $(\mathrm{C} 0)$ & Cloud $(\mathrm{C} 1)$ & Cloud $(\mathrm{C} 2)$ & Cloud $(\mathrm{C} 3)$ \\
\hline Mean & -1.35 & -1.84 & 0.30 & 0.19 & 0.32 & 0.17 & 0.49 \\
Std & 0.32 & 0.14 & 0.12 & 0.27 & 0.27 & 0.27 & 0.26 \\
\hline
\end{tabular}

method while assuming a constant $F$ is also evident when comparing to the cloud feedback assessed using an independent histogram method (e.g., see Fig. 4a of Zelinka et al. 2012; Zhou et al. 2013).

\section{Stratospheric effect}

Stratospheric temperature and water vapor variations both result in TOA radiation flux change. Many of the CMIP3 models project a substantial stratospheric moistening despite a simultaneous cooling in global warming experiments. However, the stratospheric contribution to $\Delta R$ is generally not included in the previous feedback analyses. This omission would be valid if 1) this contribution were negligible or 2) this contribution could be estimated a priori (i.e., considered to be a fast-stabilizing "stratospheric adjustment" of radiative forcing $F$ ). However, as shown below, neither condition is met in transient climate change.

Using the radiative kernels, the stratospheric temperature and water vapor effects on the OLR are explicitly estimated according to Eq. (2). Figure 2 shows that both effects generally reduce the OLR during global warming and thus act as positive feedbacks. On

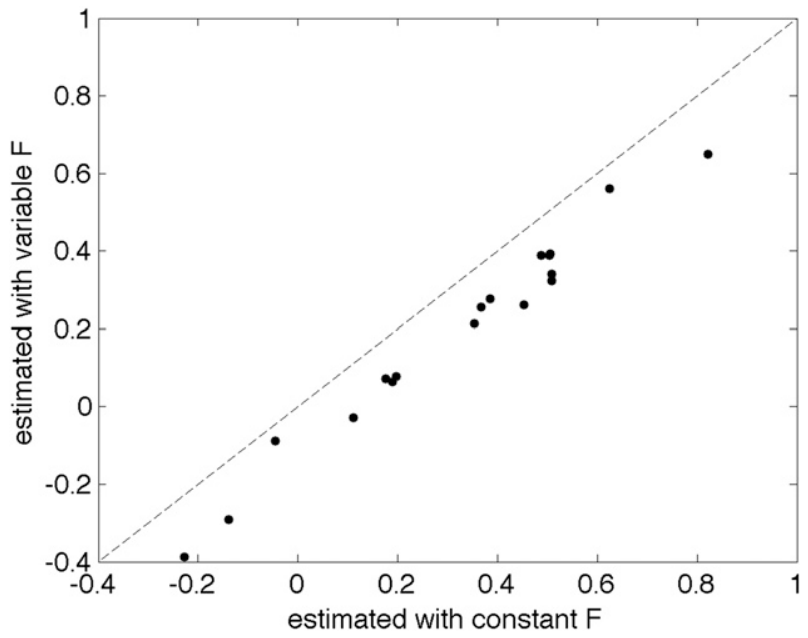

FIG. 3. Longwave cloud feedback calculated using the CFA method. The feedback strength calculated when forcing $F$ assumed to be constant across different models is compared to that calculated when $F$ is estimated for each model. average, the temperature effect is 3 times larger than the water vapor effect (see Table 1). When combined they render a multimodel mean overall stratospheric effect of $0.3 \mathrm{~W} \mathrm{~m}^{-2} \mathrm{~K}^{-1}$, with an intermodel standard deviation of $0.12 \mathrm{~W} \mathrm{~m}^{-2} \mathrm{~K}^{-1}$. Although each stratospheric effect is less than their tropospheric counterpart, the overall stratospheric effect has an intermodel spread comparable to the overall tropospheric feedback and a multimodel mean magnitude greater than the cloud feedback (see Fig. 4 and Table 2). This is because, as pointed out in previous studies (e.g., Ingram 2013; Held and Shell 2012), tropospheric temperature and water vapor feedbacks are strongly anticorrelated (a correlation coefficient of -0.87 is obtained here) due to the largely invariant relative humidity, while the stratospheric effects are not (a correlation coefficient of 0.07 here).

As the overall stratospheric effect is dominated by the temperature effect, it is important to determine what gives rise to the temperature change. If it is mainly due to $\mathrm{CO}_{2}$ or other prescribed greenhouse gases, then it may be considered part of forcing; however, if it is due to water vapor or other model-predicted greenhouse gases, it is a feedback that may vary in different models and thus cannot be assumed to be a constant forcing adjustment. Correlations between the partial OLR changes are examined: the stratospheric temperature effect on OLR, $\Delta R_{T_{\mathrm{st}}}$, is not significantly correlated with the forcing $F$ (a correlation coefficient of 0.31 ), the surface temperature change $(0.24)$, or the stratospheric water

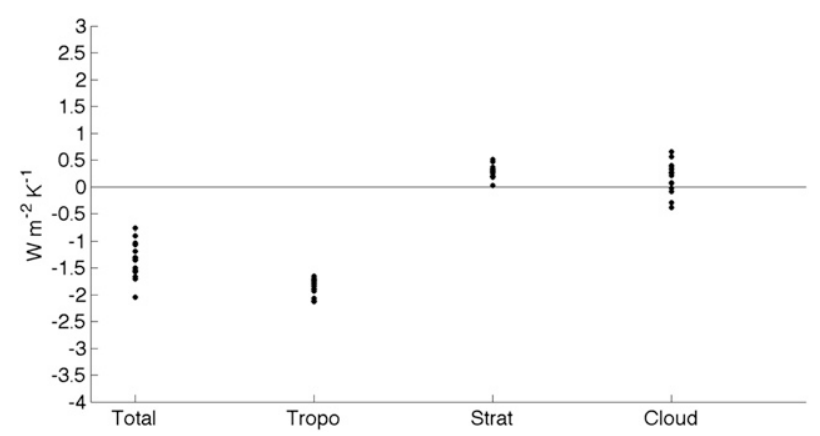

FIG. 4. Longwave climate feedbacks. Overall stratospheric effect (Strat) is compared to overall tropospheric noncloud feedback (Tropo) and cloud feedback. 
vapor effect $\Delta R_{W_{\mathrm{st}}}(0.10)$. This suggests that the stratospheric temperature changes in these models result from multiple causes encompassing both forcing and feedback. In fact, it has been recognized that multiple causes may drive stratospheric cooling (e.g., Ramaswamy et al. 1992; Forster and Shine 1999; Polvani and Solomon 2012); possible candidates include $\mathrm{CO}_{2}$ (forcing), water vapor (feedback), and ozone (forcing or feedback, depending on whether ozone change is prescribed or predicted). Particularly with regard to water vapor, Forster and Shine estimate that it may enhance the $\mathrm{CO}_{2}$-driven historical cooling by $40 \%$.

Interestingly, when the CFA method is used to estimate the cloud feedback, omitting stratospheric terms $\left(\Delta R_{T_{\mathrm{st}}}\right.$ and $\left.\Delta R_{W_{\mathrm{st}}}\right)$ in Eq. (5) introduces little error (see Table 2, case C2). This is because the clear-sky and all-sky stratospheric effects largely cancel. However, if cloud feedback is simply estimated as a residual term in Eq. (3), neglecting the stratospheric terms would result in a noticeable overestimate in cloud feedback strength (Table 2, case C3). This confirms that the CFA method is better than using solely Eq. (3) for estimating cloud feedback (Soden et al. 2008; Shell et al. 2008). Note, however, that the latter was used in earlier studies (e.g., Soden and Held 2006) and sometimes has to be adopted in analyzing observational data when clear-sky data are not available due to sampling limitations.

\section{Discussion}

The above analysis shows that use of correct forcing and inclusion of stratospheric effects are crucial for properly diagnosing the longwave climate feedbacks in transient climate change.

It needs to be cautioned that even though greenhouse gas concentrations are prescribed in scenario experiments, the radiative forcing felt by each model may differ due to model discrepancies in water vapor and cloud. When diagnosing climate feedbacks, this effect needs to be properly taken into consideration. Otherwise, model differences in surface warming projection would be solely attributed to, and thus overestimate, their sensitivity differences. A method is proposed here to first estimate the clear-sky forcing from a linear $\triangle \mathrm{OLR}$ decomposition and then estimate the all-sky forcing using an empirical relationship. This takes advantage of the fact that the nonlinearity effect is rather small in the clear-sky $\triangle$ OLR decomposition when forcing is accurately known, and removes, by design, the large residual terms in the all-sky $\triangle$ OLR decomposition that exist when intermodel variation in forcing is not accounted.

Some caveats are noted regarding the forcing treatment. The empirical ratio between the clear- and all-sky forcing [Eq. (6)] is subject to uncertainty. However, the uncertainty in this ratio, in a fractional sense, is unlikely to be as large as the uncertainty in $F$, which, from the above analysis, may be on the order of $100 \%$. So, $\left(F^{\mathrm{clr}}-F\right)$, and thus the cloud feedback assessed with the CFA method [see Eq. (5)], should be mainly affected by the uncertainty in $F$. Another caveat of attributing the residual term in Eq. (5) completely to forcing variation across the models is that possible kernel errors are ignored. However, it has been shown that kernel errors do not seem to induce errors of more than a few percent in the global mean feedbacks (e.g., Soden et al. 2008). And tests show that the clear-sky OLR in unforced climate variations can be well reproduced using temperature and water vapor kernels (e.g., Huang et al. 2007; see appendix B for more discussion). The evidence suggests that forcing uncertainty is a major cause of the residual in Eq. (5). Apparently a more robust method to assess the forcing uncertainty is to faithfully compute the forcing for each model in each scenario experiment using the partial radiative perturbation technique, although this incurs much computation. Meanwhile, developing and applying model-dependent kernels should improve feedback analysis as well (e.g., Sanderson and Shell 2012).

The stratosphere plays an important role in determining the transient variations of the OLR. Most GCMs project a stratospheric moistening despite the simultaneous stratospheric cooling in global warming experiments. Both stratospheric temperature and water vapor effects reduce the OLR and thus the overall stratospheric effect acts as a positive "feedback" in global warming. This effect has a magnitude greater than the longwave cloud feedback and an uncertainty comparable to that of the overall tropospheric feedback. Hence, it is important to recognize the stratospheric contribution to the overall climate sensitivity and its uncertainty. While the stratospheric water vapor change clearly acts as a feedback mechanism that links to surface temperature change (e.g., through convective activity; Anderson et al. 2012), it is more ambiguous whether the temperature change is due to forcing species such as $\mathrm{CO}_{2}$ or feedback species such as water vapor and ozone. The experiment analyzed here indicates that it is a mixture of both. More studies are required to attribute the stratospheric temperature change and its radiative impact.

It is important to note that ignoring the intermodel forcing variation and/or the stratospheric effect may result in biases in cloud feedback if it is not independently assessed but rather estimated as a residual term. The multimodel mean longwave cloud feedback, $0.19 \mathrm{~W} \mathrm{~m}^{-2} \mathrm{~K}^{-1}$, estimated here with both effects accounted for, is much less than an often cited number, 
$0.5 \mathrm{~W} \mathrm{~m}^{-2} \mathrm{~K}^{-1}$ (e.g., Zelinka and Hartmann 2010; Soden and Vecchi 2011); and 4 out of 18 models here have a negative longwave cloud feedback. Note that the experiment analyzed here and the experiments based on which other numbers are reported in the literature (e.g., Zelinka and Hartmann 2010; Soden and Vecchi 2011; Zelinka et al. 2012) are all different. It is likely the model ensemble mean and spread of this quantity differ with respect to experiment (e.g., equilibrium versus transient; $\mathrm{CO}_{2}$ forcing versus other types of forcing) and ensemble (e.g., CMIP3 vs CMIP5). Nevertheless, the results here and the recent results of others [e.g., the estimate of Zelinka et al. (2012) based on cloud property histogram] indicate that there is, in fact, no consensus in terms of the sign of the longwave cloud feedback among the GCMs. Even though rise of cloud top as proposed by Zelinka and Hartmann (2010) is a valid hypothesis that would induce positive cloud feedback, changes in cloud fraction and other properties may result in a negative feedback. More studies are still required to understand how clouds modify global warming, even with regard to the longwave aspect alone.

Acknowledgments. I thank Brian Soden, Isaac Held, Andrew Dessler, and John Dykema, whose comments have helped improve this paper. I also thank Mark Zelinka and an anonymous reviewer for their comments and helpful suggestions. I acknowledge Karen Shell for making her radiative kernel data available online. I acknowledge the modelling groups, the Program for Climate Model Diagnosis and Intercomparison, and the WCRPs Working Group on Coupled Modelling for their roles in making available the WCRP CMIP3 multimodel dataset.

\section{APPENDIX A}

\section{Climate Sensitivity and Feedbacks}

Climate sensitivity, $S$, is conventionally expressed as the global mean surface temperature change, $\Delta T_{s}$, in the unit of $\mathrm{K}$, in response to a certain amount of radiative forcing, $F$, which often takes a value of about $4 \mathrm{~W} \mathrm{~m}^{-2}$ and corresponds to the global mean OLR change due to doubling $\mathrm{CO}_{2}$. The usefulness of this concept is rooted in the approximately linear relationship between the global mean $\Delta T_{s}$ and global mean $F$ that is observed in GCM experiments (Ramaswamy et al. 2001). If one starts from an equilibrium climate state, imposes a radiative perturbation $F$, and lets the system re-equilibrate, at the end state the TOA radiation energy budget reinstates a balance so that the net radiation change is zero. The radiation flux change that balances $F$ results from the adjustments of various variables of the climate system, including surface temperature $\left(T_{s}\right)$, atmospheric temperature $(T)$, water vapor $(W)$, clouds $(C)$, surface albedo $(A)$, etc. Making use of the linear relationship between $F$ and $\Delta T_{s}$, one can write an equation anchored on $T_{s}$,

$$
\Delta R=F+S^{-1} \Delta T_{s}=0,
$$

so that the sensitivity of the system is measured by a single parameter $S$ which can be determined from $F$ and $\Delta T_{s}$ in a GCM experiment. Apparently $S$ depends on the details of the adjustment of the system.

Independently, the radiation flux can be considered as a function of the above variables: $R=R\left(T_{s}, T, W\right.$, $C, A, \ldots)$, so one can decompose the total change in net flux $\Delta R$ using a Taylor expansion series:

$$
\begin{aligned}
\Delta R-F= & \frac{\partial R}{\partial T_{s}} \Delta T_{s}+\frac{\partial R}{\partial T} \Delta T+\frac{\partial R}{\partial W} \Delta W+\frac{\partial R}{\partial C} \Delta C \\
& +\frac{\partial R}{\partial A} \Delta A+\operatorname{Res} \\
= & \left(\frac{\partial R}{\partial T_{s}}+\frac{\partial R}{\partial T}\right) \Delta T_{s}+\frac{\partial R}{\partial T}\left(\Delta T-\Delta T_{s}\right)+\frac{\partial R}{\partial W} \Delta W \\
& +\frac{\partial R}{\partial C} \Delta C+\frac{\partial R}{\partial A} \Delta A+\text { Res. }
\end{aligned}
$$

The first four terms on the RHS account for the change in TOA radiation caused by the Planck effect of vertically homogeneous temperature change $\left(\Delta R_{P}\right)$, by the Planck effect of vertically inhomogeneous temperature change (lapse rate change) $\left(\Delta R_{L}\right)$, by the atmospheric opacity effect of water vapor change $\left(\Delta R_{W}\right)$, by opacity effect of cloud change $\left(\Delta R_{C}\right)$, and the by surface albedo change $\left(\Delta R_{A}\right)$, in order. Note that in a single-column atmosphere $\Delta T, \Delta W$, and $\Delta C$ represent changes in the vertical profiles of these variables and thus are vectors. So each of these terms is indeed the inner product of a radiative sensitivity kernel vector $(\partial \mathbf{R} / \partial X)$ and a climate response vector $(\Delta \mathbf{X})$. For the sake of simplicity, however, let us keep denoting them as if they were scalars.

From Eqs. (A1) and (A2), it is obvious that when the higher-order residual term Res is neglected,

$$
\begin{aligned}
S= & \left\langle\left(\frac{\partial R}{\partial T_{s}}+\frac{\partial R}{\partial T}\right) \Delta T_{s}+\frac{\partial R}{\partial T}\left(\Delta T-\Delta T_{s}\right)\right. \\
& \left.+\frac{\partial R}{\partial W} \Delta W+\frac{\partial R}{\partial C} \Delta C+\frac{\partial R}{\partial A} \Delta A\right\rangle^{-1}\left\langle\Delta T_{s}\right\rangle .
\end{aligned}
$$


Or this can be simplified as

$$
S^{-1}=\lambda_{P}+\lambda_{L}+\lambda_{W}+\lambda_{C}+\lambda_{A} .
$$

Here, $\langle\cdot\rangle$ denotes global average. Also,

$$
\lambda_{X}=\frac{\left\langle\Delta R_{X}\right\rangle}{\left\langle\Delta T_{s}\right\rangle} .
$$

These terms are referred to as Planck feedback, lapse rate feedback, water vapor feedback, cloud feedback, and albedo feedback, respectively. The first term

$$
\lambda_{P}=\left\langle\Delta R_{P}\right\rangle\left\langle\Delta T_{s}\right\rangle^{-1}=\left\langle\left(\frac{\partial R}{\partial T_{s}}+\frac{\partial R}{\partial T}\right) \Delta T_{s}\right\rangle\left\langle\Delta T_{s}\right\rangle^{-1}
$$

is often called the Planck damping rate, the inverse of which measures the vertically homogeneous temperature change that would be needed to damp a given radiative forcing just by the Planck effect.

Now it is clear that the climate sensitivity, either in a GCM or in nature, can be diagnosed as the Planck damping rate being modified by feedbacks. And the sensitivity difference (uncertainty) among GCMs can be attributed to their feedback discrepancy, and, to a less extent, to their Planck damping rate discrepancy ( $\lambda_{P}$ depends on horizontal $\Delta T_{s}$ pattern and thus may differ among the models).

Although Eq. (A1) is established with regard to equilibrium climate change, a linear relationship is also noticed between transient changes in $T_{s}$ and $R$ (Gregory et al. 2004). Moreover, the breakdown of $\Delta R$ in Eq. (A2) is valid in general, regardless of equilibrium or transient climate change. So one can use the same equations as above to define a "transient climate sensitivity" parameter.

Furthermore, as radiation energy naturally consists of spectral components, the feedbacks can be divided into longwave (terrestrial) and shortwave (solar) radiation components. The major feedback factors that influence the longwave radiation include surface temperature, atmospheric temperature, atmospheric water vapor and other greenhouse gases, and clouds.

\section{APPENDIX B}

\section{The Kernel Technique}

The kernel technique (Soden et al. 2008; Shell et al. 2008) is a widely accepted method for analyzing feedback strength $\Delta R_{X}$. In this method, a noncloud feedback is approximated by multiplying an a priori calculated first-order (linear) sensitivity kernel $\partial R / \partial X$ and a climate response $\Delta X$ (either model-simulated or observed):

$$
\Delta R_{X}=\frac{\partial R}{\partial X} \Delta X
$$

and the cloud feedback can be assessed by the residual change in $R$ :

$$
\Delta R_{C}=\Delta R-F-\sum_{X} \frac{\partial R}{\partial X} \Delta X .
$$

If clear-sky radiation flux is available, one obtains a second equation:

$$
0=\Delta R^{\mathrm{clr}}-F^{\mathrm{clr}}-\sum_{X} \Delta R_{X}^{\mathrm{clr}} .
$$

Combining Eqs. (B2) and (B3) yields

$\Delta R_{C}=\left(\Delta R-\Delta R^{\mathrm{clr}}\right)-\sum_{X}\left(\frac{\partial R}{\partial X}-\frac{\partial R^{\mathrm{clr}}}{\partial X}\right) \Delta X-\left(F-F^{\mathrm{clr}}\right)$.

Soden et al. (2008) advocate using Eq. (B4) rather than Eq. (B2) for computing the cloud feedback in order to reduce the impact of the uncertainty in $F$. As $R-R^{\text {clr }}$ is known as "cloud forcing," this method is called cloud forcing adjustment (CFA) method. Note, however, that the $F$ and $F^{\text {clr }}$ values still need to be known beforehand.

One source of uncertainty in the feedbacks assessed using the above equations is the nonlinear terms neglected in the decomposition of $\Delta R$ using Eq. (A2). This uncertainty, however, is largely constrained in the case of longwave radiation, especially under the clear-sky condition, because the nonlinear kernels involving crosslayer climate responses $\left(\partial^{2} R / \partial X_{i} \partial X_{j}\right.$, with $i$ and $j$ indicating different vertical levels) are usually small. This can be verified, for instance, by calculating the secondand higher-order kernels with a standard atmospheric profile (e.g., Huang et al. 2007) and results from the nature of the nonscattering radiative transfer of the longwave radiation. From a spectral perspective, it is well known that monochromatic outgoing radiation mainly emerges from and thus is mostly sensitive to a finite " $\tau=1$ " layer, where the optical depth $\tau$ measured from the TOA to this height is roughly 1 . This means the cross-layer nonlinear terms cannot be large even though cross-layer climate responses themselves can be highly correlated. This condition may break down when nonopaque cloud (e.g., thin cirrus clouds) layers exist and monochromatic $R$ becomes sensitive to multiple layers, or when the overall atmospheric absorption is weak 
(e.g., for solar radiation, in the region where water vapor loading is very low) and a $\tau=1$ layer does not exist. In the former case, as the cross-layer terms would involve cloud, it is reasonable to attribute this nonlinear effect as part of the cloud feedback that is assessed as a residual (as done in this paper). The latter case may account for less accurate shortwave water vapor feedback assessment based on the kernel technique, although the overall magnitude of this feedback is far less than the longwave water vapor feedback.

Another source of uncertainty comes from the uncertainty in the linear kernels themselves, which may result from radiation code errors as well as dependence of kernel values on atmospheric states. However, with regard to code errors, Huang et al. (2007, see their Tables 1 and 2) show that band model-simulated kernels differ from those simulated by benchmark line-by-line model-simulated kernels by no more than a few percent; Soden et al. (2008, their Table 3) show that the discrepancy between multiple band models is of the same small magnitude. With regard to dependency on the atmosphere, Soden et al. (2008, see their Fig. 7) show that the global mean feedbacks calculated from different sets of kernels also agree with each other remarkably well. Furthermore, Sanderson and Shell (2012) explicitly account for intermodel differences in the kernels, but their result (e.g., see their Fig. 5) shows that the resulting differences in the global mean longwave feedbacks are very small. Finally, one can estimate the impact of the kernel errors by examining the extent to which the clear-sky OLR anomalies can be explained by using the temperature and water vapor kernels in an unforced climate model integration (prescribed with fixed well-mixed greenhouse gas concentrations). When a single set of kernels is used for multiple arbitrarily selected models, it is found that no less than $70 \%$ of the global annual mean OLR anomalies are reproduced by using the kernels. In summary, the evidence suggests that kernel errors do not severely impact the diagnosed feedbacks.

\section{REFERENCES}

Anderson, J. G., D. M. Wilmouth, J. B. Smith, and D. S. Sayres, 2012: UV dosage levels in summer: Increased risk of ozone loss from convectively injected water vapor. Science, $\mathbf{3 3 7}$, 835-839.

Forster, P. M. D., and K. P. Shine, 1999: Stratospheric water vapour changes as a possible contributor to observed stratospheric cooling. Geophys. Res. Lett., 26, 3309-3312.
Gregory, J. M., and Coauthors, 2004: A new method for diagnosing radiative forcing and climate sensitivity. Geophys. Res. Lett., 31, L03205, doi:10.1029/2003GL018747.

Hansen, J., M. Sato, and R. Ruedy, 1997: Radiative forcing and climate response. J. Geophys. Res., 102 (D6), 6831-6864.

Held, I. M., and K. M. Shell, 2012: Using relative humidity as a state variable in climate feedback analysis. J. Climate, 25, 25782582.

Huang, Y., 2013: A simulated climatology of spectrally decomposed atmospheric infrared radiation. J. Climate, 26, 1702-1715.

- , V. Ramaswamy, and B. Soden, 2007: An investigation of the sensitivity of the clear-sky outgoing longwave radiation to atmospheric temperature and water vapor. J. Geophys. Res., 112, D05104, doi:10.1029/2005JD006906.

— S. Leroy, P. J. Gero, J. Dykema, and J. Anderson, 2010: Separation of longwave climate feedbacks from spectral observations. J. Geophys. Res., 115, D07104, doi:10.1029/ 2009JD012766.

Ingram, W., 2013: A new way of quantifying GCM water vapour feedback. Climate Dyn., 40, 913-924.

Polvani, L. M., and S. Solomon, 2012: The signature of ozone depletion on tropical temperature trends, as revealed by their seasonal cycle in model integrations with single forcings. J. Geophys. Res., 117, D17102, doi:10.1029/2012JD017719.

Ramaswamy, V., M. D. Schwarzkopf, and K. P. Shine, 1992: Radiative forcing of climate from halocarbon-induced global stratospheric ozone loss. Nature, 355, 810-812.

_- and Coauthors, 2001: Radiative forcing of climate change. Climate Change 2001: The Scientific Basis, J. T. Houghton et al., Eds., Cambridge University Press, 349-416.

Sanderson, B. M., and K. M. Shell, 2012: Model-specific radiative kernels for calculating cloud and noncloud climate feedbacks. J. Climate, 25, 7607-7624.

Shell, K. M., J. T. Kiehl, and C. A. Shields, 2008: Using the radiative kernel technique to calculate climate feedbacks in NCAR's Community Atmospheric Model. J. Climate, 21, 2269-2282.

Soden, B. J., and I. M. Held, 2006: An assessment of climate feedbacks in coupled ocean-atmosphere models. J. Climate, 19, 3354-3360.

— , and G. A. Vecchi, 2011: The vertical distribution of cloud feedback in coupled ocean-atmosphere models. Geophys. Res. Lett., 38, L12704, doi:10.1029/2011GL047632.

_ I. M. Held, R. Colman, K. M. Shell, J. T. Kiehl, and C. A. Shields, 2008: Quantifying climate feedbacks using radiative kernels. J. Climate, 21, 3504-3520.

Solomon, S., D. Qin, M. Manning, Z. Chen, M. Marquis, K. Averyt, M. Tignor, and H. L. Miller Jr., Eds., 2007: Climate Change 2007: The Physical Science Basis. Cambridge University Press, 996 pp.

Zelinka, M. D., and D. L. Hartmann, 2010: Why is longwave cloud feedback positive? J. Geophys. Res., 115, D16117, doi:10.1029/ 2010JD013817.

_ S. A. Klein, and D. L. Hartmann, 2012: Computing and partitioning cloud feedbacks using cloud property histograms. Part I: Cloud radiative kernels. J. Climate, 25, 3715-3735.

Zhou, C., M. D. Zelinka, A. E. Dessler, and P. Yang, 2013: An analysis of the short-term cloud feedback using MODIS data. J. Climate, 26, 4803-4815. 